\title{
One week of exposure to sunlight induces progerin expression in human skin
}

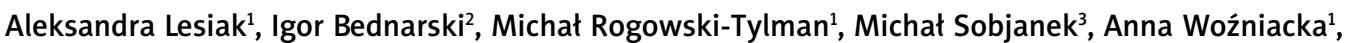 \\ Marian Danilewicz ${ }^{4}$, Anthony Young ${ }^{5}$, Joanna Narbutt ${ }^{1}$
}

${ }^{1}$ Department of Dermatology, Medical University of Lodz, Lodz, Poland

2Department of Dermatology, Pediatric Dermatology and Dermatological Oncology, Medical University of Lodz, Lodz, Poland ${ }^{3}$ Department of Dermatology, Venereology and Allergology, Medical University of Gdansk, Gdansk, Poland ${ }^{4}$ Department of Pathology, Medical University of Lodz, Lodz, Poland ${ }^{5}$ St John's Institute of Dermatology, King's College London, London, UK

Adv Dermatol Allergol 2017; XXXIV (6): 629-631 DOI: https://doi.org/10.5114/pdia.2016.62416

Solar ultraviolet radiation (UVR) causes sunburn, suppression of acquired immunity, photoageing and skin cancer. Erythema and immunosuppression are acute effects whereas cancers and photoageing develop many years later. Skin photodamage is a complex sequence of phenomena that lead to structural and functional changes which impair the skin's adaptive and barrier functions. The skin is also important in social interactions and the development of strategies against skin photodamage plays a significant role in dermatological research and public health [1-4]. Ultraviolet radiation damages dermal collagen by inhibiting procollagen biosynthesis and the promotion of matrix-metalloproteinase (MMP) expression. Single exposures to erythemal UVR cause a complete loss of procollagen synthesis, which persists for $24 \mathrm{~h}$ and with recovery at 48-72 $\mathrm{h}$ [4]. Studies on the genetic disorder Hutchinson-Gilford syndrome (HGPs; progeria) have shown that the overexpression of progerin, a mutated form of laminin A (main nuclear matrix protein) leads to nuclear deformation and impairment of cell proliferation. The consequences of such deformation are an alteration of histone patterns, abnormal chromatin remodeling, reduction of DNA repair processes, increase in telomere shortening and overexpression of p53, all of which initiate early senescence [5]. In young healthy subjects, the progerin expression is observed in nuclei in the papillary dermis and in the basement membrane zone, while in elderly subjects it is also found in the reticular dermis [5]. However, there is the lack of literature on the influence of UVR on progerin expression in healthy subjects. Thus, we aimed to assess progerin expression in normal human skin exposed to UVR in real life to determine whether progerin expression may be considered as a molecular marker of skin dysregulation.
The Ethics Committee of the Medical University of Lodz approved the study and each participant gave written informed consent. The study was done according to the Declaration of Helsinki and subjects were recruited by the Dermatology Department of the Medical University of Lodz, Poland.

The study population comprised 30 healthy Caucasians from whom we took $4 \mathrm{~mm}$ diameter skin punch biopsies from the outer upper arm. All subjects were recruited by the Dermatology Department of the Medical University of Lodz, Poland. Exclusion criteria were a history of skin disease (including skin cancer), taking photosensitizing medication and sunbed/holiday sun exposure within the previous 4 months. The subjects were divided into two groups. Group 1 included 15 elderly people with strong clinical evidence of photoaged skin (7 female, 8 male, mean age: $64.1 \pm 13.1$ years; phototype $\|-n=7$ or III $-n=8)$. Group 2 comprised 15 healthy young adults (7 female, 8 male, mean age: $24.1 \pm 4.8$ years, phototype $\|-n=6$ or $\| I-n=9$ ) who participated in 6-day sun holiday in Tenerife (Canary Islands) Spain $\left(28^{\circ} \mathrm{N} 16^{\circ} \mathrm{W}\right)$ in March 2011. The volunteers were not instructed on sunscreen use, which was not monitored to avoid any influence on their photoprotection habits. Participants had approximately $5 \mathrm{~h}$ of sun exposure every day between 09:00 to 17:00 local time. Two biopsies were taken from group 2; one $24 \mathrm{~h}$ before the holiday (group 2a) and $24 \mathrm{~h}$ after returning (group $2 b$ ).

Skin specimens were frozen at $-80^{\circ} \mathrm{C}$ and stored until analysis. Progerin expression was assessed by immunohistochemical staining with a mouse monoclonal antibody (1 : 10 dilution, 66587 Abcam, Cambridge, UK). Histological morphometry was performed by computerized image analysis using a Pentagram graphics tablet (Indeo

Address for correspondence: Igor Bednarski, Department of Dermatology, Pediatric Dermatology and Dermatological Oncology, Medical University of Lodz, 1/5 Kniaziewicza St, 91-347 Lodz, Poland, fax: +48 42651 10 72, e-mail: igorbednarskiv@gmail.com Received: 28.06.2016, accepted: 30.08.2016. 
Table 1. Percentage of progerin positive nuclei in study groups

\begin{tabular}{ccccc}
\hline Protein & $\begin{array}{c}\text { Group 1 } \\
\text { (older subjects) }\end{array}$ & $\begin{array}{c}\text { Group 2a } \\
\text { (younger subjects pre-holiday) }\end{array}$ & $\begin{array}{c}\text { Group 2b } \\
\text { (younger subjects post-holiday) }\end{array}$ & $P$-value \\
\hline Progerin (\%) & $1.55 \pm 0.61$ & $0.00 \pm 0.00$ & $0.97 \pm 0.39$ & $0.001^{1}$ \\
& & & $<0.001^{2}$ \\
& & & $<0.03^{3}$ \\
\hline
\end{tabular}

${ }^{2}$ Between groups $2 b$ and $2 a,{ }^{2}$ between groups $2 a$ and group $1,{ }^{3}$ between groups $2 b$ and group 1.
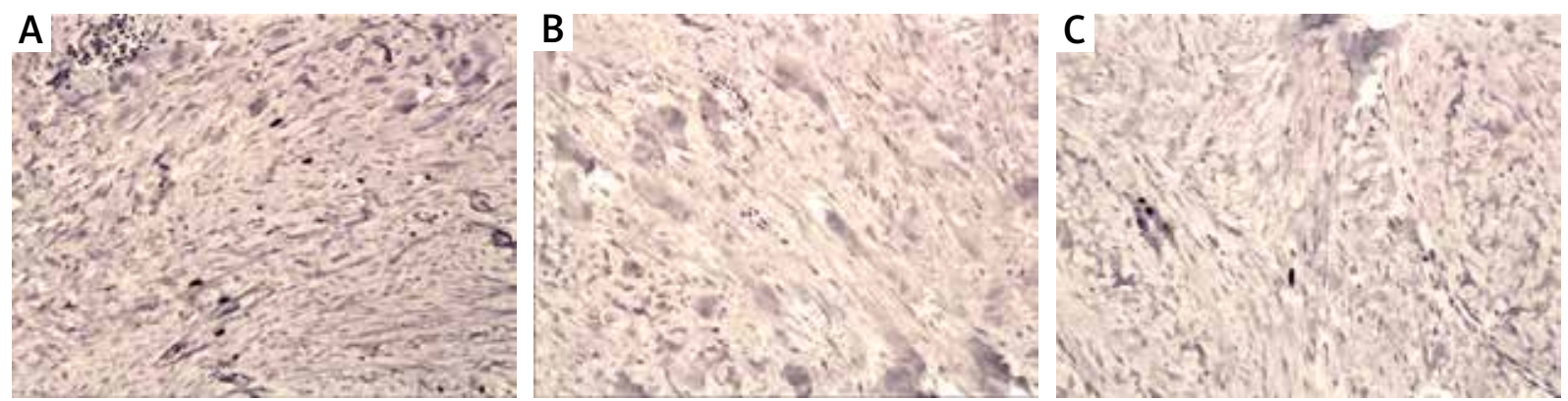

Figure 1. A - Nuclear immunoexpression of progerin in dermal fibroblasts in group 1. Mag. 100x. B - Negative staining for progerin in a control case (group 2a). Mag. 100x. C - Weak nuclear immunoexpression of progerin in dermal fibroblasts in group $2 \mathrm{~b}-$ after coming back from Tenerife. Mag. 100x

Fast card with true-colour real-time frame grabber produced by Indeo (Taiwan), and colour camera Panasonic (Japan) coupled to a Carl Zeiss microscope (Germany)). This system was programmed (MultiScan 8.08 software, produced by Computer Scanning Systems, Poland) to calculate the number of immunopositive cells (semiautomatic function). The microscopic images were saved serially and then quantitative examination was performed. The percentage of progerin positive cells was estimated in the dermis by counting 100 cells in ten monitor fields $\left(0.029 \mathrm{~mm}^{2}\right.$ each), with a total assessment of 1000 cells per sample.

The results were compared by the Student's t-test for independent samples after an evaluation of normality and homogeneity of variances with Levene's test. Additionally the Mann-Whitney $U$ test was used where appropriate.

Progerin was not present in young volunteers prior to sun exposure (group 2a). Its expression was exclusively nuclear in the other groups, mainly in dermal fibroblasts, and very rarely in keratinocytes. Table 1 shows that the expression of progerin was greatest in group 1 (mean value: $1.55 \pm 0.61$ and was statistically higher than in group 2 a (mean value: $0.00 \pm 0.0 ; p<0.001)$ and group $2 b(0.97$ $\pm 0.39 ; p<0.03)$. There was more progerin in group $2 b$ than $2 \mathrm{a}(p=0.001)$. Figure 1 shows representative staining.

Progerin is highly expressed in HGP patients $[6,7]$ and a recent study has shown that progerin is also expressed in normal dermal ageing [7]. However, there are discrepancies in the literature. Mc Clintock et al. [8] did not find any association between progerin expression and age, but UVR exposure was not taken into account. It has been postulated that nuclear accumulation of progerin may be induced by UVR and involved in photoageing mechanisms. An in vitro study by Takeuchi and Rünger [9] showed that fibroblasts from older donors exposed to UVR accumulated more nuclear progerin than neonatal fibroblasts. It has also been shown that exposure to UVA, but not UVB, induces progerin expression in neonatal and aged fibroblasts. Progerin intensity and distribution differ with age. In our study, its distribution was similar in the elderly people and those with 1 week's exposure to UVR. These data suggest a new pathway of photoageing. We have shown that 1 week's sun exposure in healthy individuals is enough to significantly increase the expression of the protein in human dermis. To our knowledge this is the first in vivo study. Our data suggest that progerin may be a molecular marker of photoageing and the inhibition of progerin pathway may be regarded as a new therapeutic approach. The increase in progerin expression after 1 week of UVR exposure in normal life conditions (holidays) also strongly supports the need of photoprotection.

\section{Acknowledgments}

The study was funded by the National Center of Science grant no. 2012/05/B/NZ5/01885 and EC Framework 7 Programme under contract No. 227020: The Impact of Climate and Environmental Factors on Personal Ultraviolet Radiation Exposure and Human Health (ICEPURE) and 
Medical University of Lodz, project no. 503/5-064-01/503-

01 and 503/1-152-01/503-01.

\section{Conflict of interest}

The authors declare no conflict of interest.

\section{References}

1. Tierney EP, Hanke CW. Recent advances in combination treatments for photoaging: review of the literature. Dermatol Surg 2010; 36: 829-40.

2. Rabe JH, Mamelak AJ, McElgunn PJS, et al. Photoaging: mechanisms and repair. J Am Acad Dermatol 2006; 55: 1-19.

3. Antoniou C, Kosmadaki MG, Stratigos AJ, Katsambas AD. Photoaging prevention and topical treatments. Am J Clin Dermatol 2010; 11: 95-102.

4. Quan T, Quin Z, Xia W, et al. Matrix degrading metaloproteinases in photoaging. J Invest Dermatol 2009; 14: 20-4.

5. Cao K, Capell B, Erdos MR, et al. A Lamin A protein isoform overexpressed in Hutchinson-Gilford progeria syndrome interferes with mitosis in progeria and normal cells. Proc Natl Acad Sci U S A 2007; 104: 4949-54.

6. Busch A, Kiel T, Heupel WM, et al. Nuclear protein import is reduced in cells expressing nuclear envelopathy-causing lamin A mutants. Exp Cell Res 2009; 315: 2373-85.

7. Scaffidi P, Misteli T. Lamin A-dependent nuclear defects in human aging. Science 2006; 312: 1059-63.

8. Mc Clintock D, Ratner D, Lokuge M, et al. The mutant form of lamin A that causes Hutchinson-Gilford progeria is a biomarker of cellular aging in human skin. PLoS One 2007; 2: e1269.

9. Takeuchi H, Rünger TM. Longwave UV light induces the aging-associated progerin. J Invest Dermatol 2013; 133: 1857-62. 